\title{
A Tool for Assessing the Text Legibility of Digital Human Machine Interfaces
}

\section{International Symposium on Resilient Cognitive Systems -- 2015 Resilience Week (RSW)}

Roger Lew

Ronald L. Boring

Thomas A. Ulrich

The INL is a

U.S. Department of Energy

National Laboratory

operated by

Battelle Energy Alliance

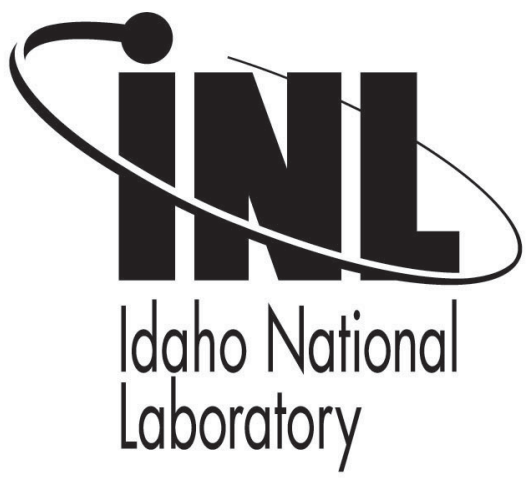

\section{August 2015}

This is a preprint of a paper intended for publication in a journal or proceedings. Since changes may be made before publication, this preprint should not be cited or reproduced without permission of the author. This document was prepared as an account of work sponsored by an agency of the United States Government. Neither the United States Government nor any agency thereof, or any of their employees, makes any warranty, expressed or implied, or assumes any legal liability or responsibility for any third party's use, or the results of such use, of any information, apparatus, product or process disclosed in this report, or represents that its use by such third party would not infringe privately owned rights. The views expressed in this paper are not necessarily those of the United States Government or the sponsoring agency. 


\section{A Tool for Assessing the Text Legibility of Digital Human Machine Interfaces}

\author{
Roger Lew \\ Virtual Technology and Design Department \\ University of Idaho \\ Moscow, ID, 83843, USA \\ rogerlew@vandals.uidaho.edu
}

\author{
Ronald L. Boring, \& Thomas A. Ulrich \\ Human Factors, Statistics, and Controls Department \\ Idaho National Laboratory \\ Idaho Falls, Idaho, 83415, USA \\ \{ronald.boring|thomas.ulrich\}@inl.gov
}

\begin{abstract}
A tool intended to aid qualified professionals in the assessment of the legibility of text presented on a digital display is described. The assessment of legibility is primarily for the purposes of designing and analyzing human machine interfaces in accordance with NUREG-0700 and MIL-STD 1472G. The tool addresses shortcomings of existing guidelines by providing more accurate metrics of text legibility with greater sensitivity to design alternatives.
\end{abstract}

Keywords-Human Factors, Text Legibility, Human Machine Interface.

\section{INTRODUCTION}

In control rooms of nuclear power plants the distributed control systems (DCSs) embedded in the control panels have grown much more usable and capable. Upgrading to modern DCSs with software based human machine interfaces (HMIs) helps to ensure the continued safe and reliable operation of the plants. Retrofitting video display units (VDUs) into existing control boards presents both practical and ergonomic challenges. On the practical side plant engineers must figure out how to make room for displays and human input devices, install them in a manner that will meet seismic requirements, and conduct the retrofit in a timely fashion. These factors must be weighed against the ergonomic consequences of the HMI's physical placement and specifications.

Unlike typical work environments consisting of seated workstations, supporting adequate ergonomics at the panels can be difficult in the control room. With sitting workstations ergonomics can be assured by providing adjustable equipment. However, designing and evaluating standing workstations with fixed displays presents challenges. The lack of adjustability results in much larger viewing distance variability from operators' eyes to the display. As the viewing distance increases the text on the display both appears smaller and becomes more difficult to read. The legibility of text in digital HMIs is of critical importance. Whether or not an operator can decipher strings of alphanumeric characters into meaningful information is dependent on several factors: the operator's visual acuity, the ambient luminance of the environment, the contrast and typography of the text, as well as the physical size and location of the text relative to the operator's eyes. From a practical standpoint, most of these influences can be treated as invariant factors. Licensed operators - much like licensed drivers- have visual acuity standards that must be met. Illuminance levels in control rooms are mandated by NUREG-0700 (see sections 12.1.2.3-1). NUREG 0700 also specifies typography guidelines, as well as luminance and contrast guidelines specifically for VDUs; (see [1], [2] for background regarding the influence of contrast and font color selection). Thus, the digital HMI designer's primary concern is to quantify the subtended visual angle of text in the operator's visual field. In other words, HMI designers must carefully consider the perceived size of text by the operators. Current NUREG-0700 guidelines pertaining to text legibility are of limited practical utility to digital HMI designers because they are intended to specify the physical size of text on control boards and not the required font size for implementing digital HMIs. While the mathematics dictating subtended angle are intuitive and straightforward, the litany of factors involved in performing such calculations for design and evaluation purposes makes controlling text size in digital environments deceptively complex. Here we present a fieldtested engineering and design tool for assessing the relative merit of alternative text display configurations.

\section{BACKGROUND}

\section{A. Minimum Viewing Angle}

NUREG-0700 [3] specifies minimum of viewing angles in minutes of $\operatorname{arc}\left(\right.$ MOA or $\left.{ }^{\hat{\gamma}}, 1^{\circ}=60^{\hat{\gamma}} 1 \mathrm{rad}=10800 / \pi^{\prime}\right)$ as $15^{\hat{\imath}}$ in guideline 12.2.1.2 for general labels, $16^{\hat{\gamma}}$ in guideline 1.3.1-4 with $20-24^{\hat{\prime}}$ preferred for reading tasks, and $15^{\hat{\prime}}$ in 1.3.1-7 for character separation. Those familiar with NUREG-0700 might recall the formula given in guideline 1.3.1-7 describing the required text height as a function of viewing distance $(d)$ and the required MOA guideline $(m a)$ :

$$
h=6.283 d(m a) / 21600 .
$$

However, this equation is not in a form to aid digital HMI designers, since they do not directly specify the physical size of fonts as part of the design process. They must specify the size of text as font point sizes (font sizes), and for crisp text, font sizes can only be specified as whole numbers. The physical height of text is the product of the font size and the height of the pixels in the display (dot pitch, pixel pitch). As a result text 
size cannot be continuously varied; but rather it can only be varied at discretized increments of the display's pixel pitch.

\section{B. An Anthropometric Model for Assessing Viewing Distance and Viewing Angle}

Current best practices recommend using population stereotypes, consisting of anthropometric characteristics of individuals falling on extreme tails of the distribution, $5^{\text {th }}$ percentile female and $95^{\text {th }}$ percentile male, to test whether a configuration is acceptable or not acceptable for these extremes. While this is effective at testing compliance to NUREG standards it leads to several less than desirable repercussions, many of which are analogous to criticisms of significance testing. Significance tests in statistics are often misapplied, arbitrary, and misinterpreted [4] 1) Population stereotypes focus designers on designing for the outliers instead of designing for the masses (the individuals in the middle of the distribution). 2) Much like setting $\alpha=0.05$ with null hypothesis significance testing; the selection of $16 \mathrm{MOA}$ for general labels is arguably arbitrary. Arditi and Cho [5] have found $10 \mathrm{MOA}$ is sufficient for legibility of random characters for individuals with normal vision (20/30 or better). 3) Its "yes" or "no" dichotomy emphasizes decision over diagnosticity. It is not practical to categorize an interface satisfying a 94.99th percentile male but not a 95th percentile male as a failure. Rather, the key should be to understanding the trade-offs between critical factors and design constraints.

Ergonomic assessments should evaluate how well technology is suited to the population of current and future operators. Here, the calculations that would normally be applied to an individual are being applied to populations. The NUREG guidelines for consoles operators stand at are based on anthropological data from MIL-STD 1472G [6]. For each sex, male and female, the mean $(\mu)$ and standard deviation $(\sigma)$ can be used to define a population stature distribution (see Figure 1) with a probability density function of:

$$
f(x) \stackrel{\text { def }}{=} \frac{e^{-(x-\mu)^{2} /(2 \sigma)^{2}}}{\sigma \sqrt{2 \pi}},
$$

and a cumulative density function of:

$$
F(x) \stackrel{\text { def }}{=} \int_{-\infty}^{x} f(x) \text {. }
$$

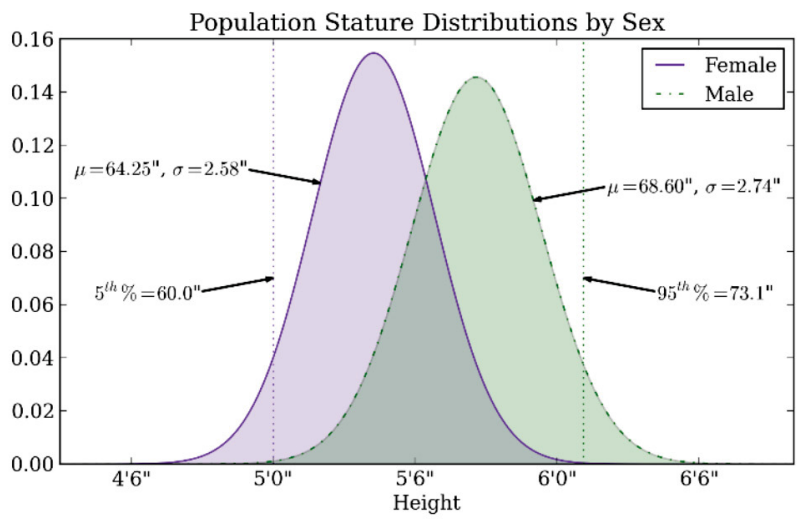

Figure 1. Stature gender distributions by sex.

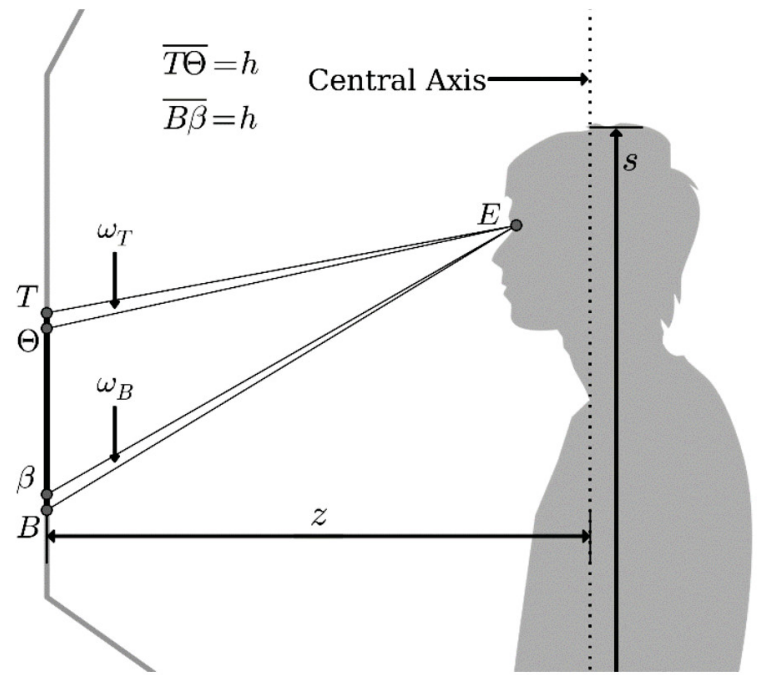

Figure 2. Anthropometric model of an operator standing in front of a digital HMI

The guideline presented in Equation 1 can be shown to be a linear approximation of the tangent identity assuming that the observer's view is perpendicular to the screen. However, this assumption is rarely met with a display at a fixed height, which may result in off-axis viewing angles that decrease the subtended angle and overall legibility. Cai, Green, Kim [7] provide empirical evidence demonstrating viewing angle systematically affects legibility. Here, an anthropometric model of a standing workstation and operator is developed without the perpendicular viewing angle assumption. Figure 2 depicts such a model. The display is represented by the segment $(\overline{T B})$ where point $B$ is the bottom of the screen and point $T$ is the top of the screen. The distance $z$ defines the horizontal distance from point $B$ to the operator's central axis. The eyepoint $E$ relative to the display is a function of $z$, the height the display from the ground, the tilt angle of the display (not depicted), the stature of the individual $(s)$, and lastly whether the individual is sitting or standing (depicted standing).

A designer may wish to know if a given font point size $(p t)$ will be rendered at a sufficient height $(h)$ to be legible on a particular display. The height of the characters on the screen is the product of the font size and the dot pitch of the display in question. Dot pitch is the reciprocal of pixel density (pixels per inch, $p p i)$. Given a speculative point size ( $p t)$ and the $p p i$ of the display under evaluation one can calculate height using,

$$
h=p t \frac{1}{p p i} .
$$

Designers need to ensure that text at this size would be legible regardless of where it is placed on the screen. To do so the model should assume the text would be placed along the horizontal edge of the screen farthest from the eye point. For brevity let's assume the bottom edge is furthest (as depicted). From $h, B$, and the screen's tilt angle, it is possible to define a point representing the top of the text on the screen. This is point $\beta$, and is defined such that the distance from $B$ to $\beta$ is $h$. We now 
have a triangle demarcated by points $B, E$, and $\beta$. Viewing angle $\left(\omega_{B}\right)$ is given by the angle $\angle B E \beta$. Then the law of cosines can be used to derive $\omega_{B}$ :

$$
\begin{aligned}
& h^{2}=\overline{E B}^{2}+\overline{E \beta}^{2}-2 \overline{E B} \overline{E \beta} \cos \left(\omega_{B}\right) \Rightarrow \\
& \omega_{B}=\operatorname{acos}\left(\frac{\overline{E B}^{2}+\overline{E \beta}^{2}-h^{2}}{2 \overline{E B} \overline{E \beta}}\right) .
\end{aligned}
$$

Likewise angle $\left(\omega_{T}\right)$ can be derived as:

$$
\omega_{T}=\operatorname{acos}\left(\frac{\overline{E T}^{2}+\overline{E \Theta}^{2}-h^{2}}{2 \overline{E T} \overline{E \Theta}}\right) .
$$

The critical viewing angle used for analysis is defined as:

$$
\omega \stackrel{\text { def }}{=} \min \left(\omega_{B}, \omega_{T}\right) \text {. }
$$

Taking the smaller viewing angle assures that the analysis is conservative. The viewing distance $(d)$ can be assessed as:

$$
d \stackrel{\text { def }}{=} \max (\overline{E T}, \overline{E B}) .
$$

Finally stature distributions can be applied to the anthropometric model to determine the portion of the population that is accommodated by the display and font size arrangement. These results are then presented in a graphical manner (see Figure 3) to identify the optimal placement of a display, as well assessing design tradeoffs between constraining variables.

\section{IMPLEMENTATION}

The tool was developed in the Python programming language and using Numpy and SciPy to handle the geometric calculations and the statistical distribution functions, and Matplotlib was used to generate the graphical output. The Flask microframework and Google's appengine for Python was used to produce a web-deployable application. Because the application runs server-side there is no need for end-users to have any specialized software or experience with Python to perform text legibility analyses. This application can be found at:

\section{http://il-legible.appspot.com/}

with the accompanying source code at:

\section{https://github.com/rogerlew/il-legible-appengine}

\section{INTERPRETING THE FIGURE}

The assumptions of the model are provided in the top left corner. To the right of the model's assumptions is a figure depicting the vertical distance from the user's eye to the farthest vertical location on the screen (either the top or bottom) as a function of stature. View angle is depicted in teal. A view angle of zero indicates the center of the screen is perpendicular to the user's gaze. Negative view angles indicate the user would be looking up at the screen, positive angles indicate the user would be looking down.
The table in the top right corner lists the font sizes used in the analysis and their corresponding physical heights in inches and millimeters.

The main figure depicts viewing angle in MOA as a function of viewing distance and font size. The isopleths indicate contours of constant font size. Viewing distance is a function of the display's placement, the horizontal distance from the display to the user, and the user's stature. In the analysis all of these factors are fixed except for stature so a monotonic relationship exists between viewing distance and stature

The green horizontal line indicates the threshold for NUREG-0700 compliance. For a given viewing distance a font size is compliant if it is above this line.

The bottom figures depict how a given font will satisfy the potential population distribution of users. The probability density functions for females and males are shaded in light gray. For each distribution the percentiles satisfied by the font size are shaded in green. The tables underneath each list the lower (L) and upper (U) bounds as well as the total $(\mathrm{T})$ percentage of the populations accommodated by the font size.

\section{CASE STUDY}

The Human Systems Simulation Laboratory (HSSL) of the Idaho National Laboratory (INL) has assisted a major U.S. utility with conducting human factors engineering (HFE) reviews and user testing workshops related to upgrading particular systems to digital interfaces [8]. One of the realities of control room modernization is that existing control boards must be modified to accommodate new displays for digital HMIs. Even when upgrading to digital systems, many existing electromechnical controls and indicators must remain in place on the control panel. Equipment that is placed in the control room must adhere to strict seismic standards and other regulatory conditions limiting available alternatives. As a consequence, the size and location of displays becomes constrained. At a formative (early stage) usability testing workshop, a 19" display was incorporated into a mock workstation design. A vendor provided Alarm Summary display contained text that was illegible to several viewers. The default text in the alarm's summary was only 10 pixels high on a monitor with a pixel density of 119 ppi. For subsequent upgrade projects the tool has been used earlier in the design process during the stage where plant engineers begin identifying what components will be removed to make room for new displays. Text legibility during this early stage affords designers with more flexibility for pursuing the upgrade and avoids the potential problem in which the allocated area is too small to support adequate text size.

\section{DISCUSSION}

Control room modernization of nuclear control rooms often has unique ergonomic challenges due to constraints imposed by existing indicators and controls. When assessing alternatives it becomes clear that in some instances a small difference, such as an inch here or there, can make the difference between success and failure. On VDUs a point in font size can make the critical difference between legibility and 
Model Assumptions

23.9" diagonal, 1920x1080, 0.01085" dot pitch 15" Bottom of Screen Height: 51.0"

Vertical Tilt: 0.0

z Distance: $28.0^{\prime \prime}\left(40^{\circ}\right.$ FOV $)$

Posture: Standing

Population: 50\% Male

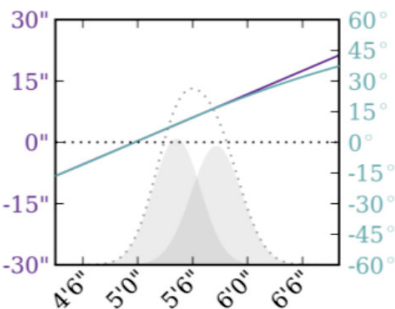

$\begin{array}{lll}10 \mathrm{Pt} & 0.108 " & 2.76 \mathrm{~mm} \\ 12 \mathrm{Pt} & 0.130^{\prime \prime} & 3.31 \mathrm{~mm} \\ 14 \mathrm{Pt} & 0.152^{\prime \prime} & 3.86 \mathrm{~mm} \\ 16 \mathrm{Pt} & 0.174 " & 4.41 \mathrm{~mm} \\ 18 \mathrm{Pt} & 0.194^{\prime \prime} & 4.96 \mathrm{~mm}\end{array}$
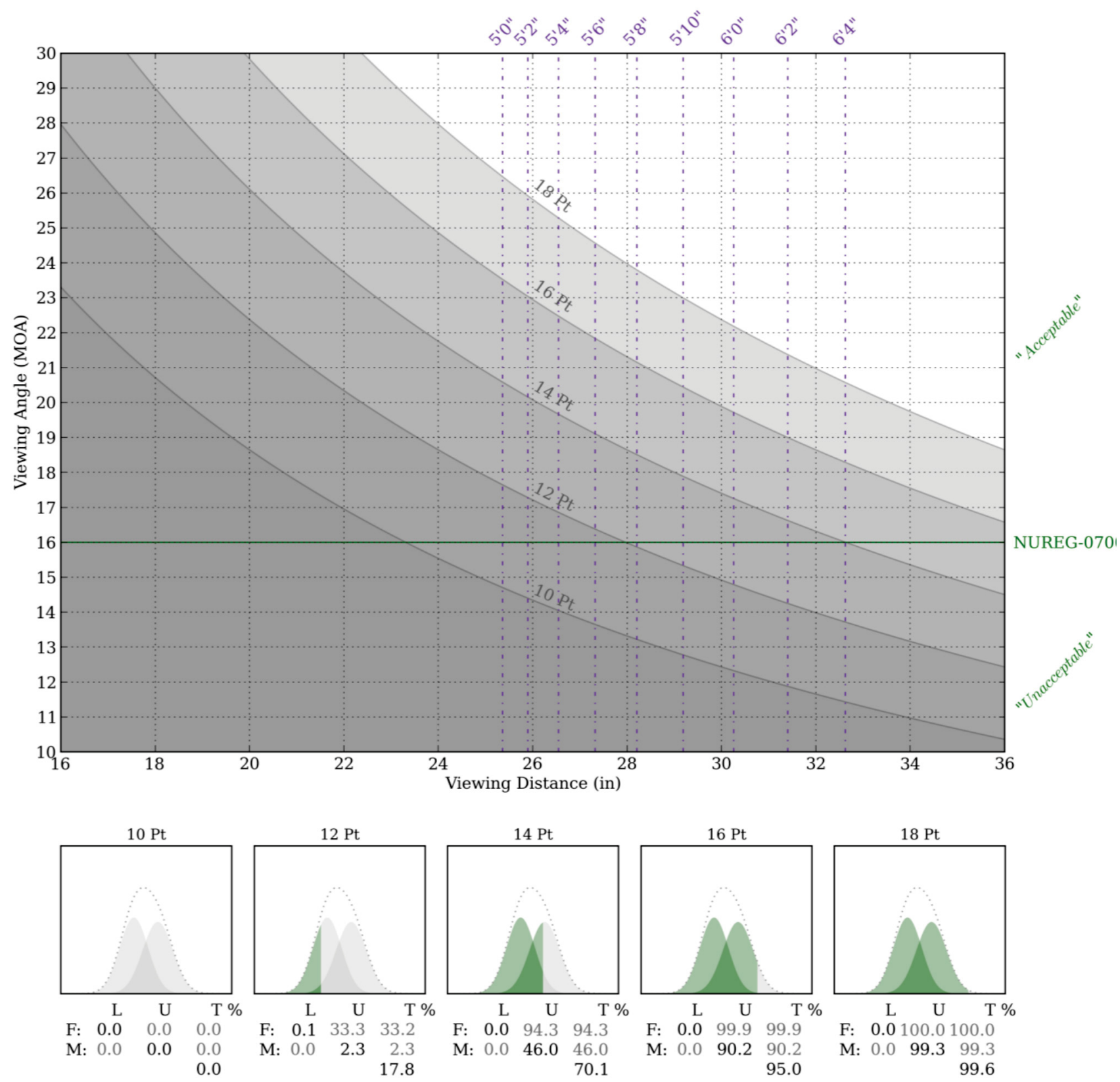

Figure 3. Graphical output of the text legibility tool for a hypothetical standing workstation with a 23.9" monitor mounted 51" above the ground and the operator standing 28 " back. See text for explanation. 
illegibility. This tool aids in the design process by allowing practitioners to assess alternatives in a systematic qualitative fashion, ensuring that both digital and analog human machine interfaces are usable and in compliance with governing safety regulations.

Users of this tool should keep in mind that estimates at offaxis viewing angles will be more conservative than the NUREG-0700 guideline because it does not assume the display is perpendicular to the operator's gaze.. If extreme off-angle viewing is expected to be commonplace, the legibility metric developed by Cai, Green, and Kim [6] should be considered. Furthermore, operators can more easily compensate for a display that is too low compared to a display that is too high. The calculations assume that operators are standing upright. In practice, taller operators have been (anecdotally) observed to lean forward to reach controls on the lower portion of control boards and to increase the legibility of both digital and analog indicators. In contrast it is more difficult for short operators to make themselves tall.

In summary, this tool (1) provides font size parameters needed by HMI designers, (2) avoids narrow focus on extreme ends of the population height, (3) accommodates for viewing angle in determining text legibility, and lastly (4) offers a more complex and accurate assessment of key human interaction with technology compared to existing guidelines.

\section{DISCLAIMER}

The software tool was developed by the first author at the University of Idaho and the source is available under the BSD 3-Clause License. Portions of this work of authorship were prepared as an account of work sponsored by an agency of the United States Government. Neither the United States
Government, nor any agency thereof, nor any of their employees makes any warranty, express or implied, or assumes any legal liability or responsibility for the accuracy, completeness, or usefulness of any information, apparatus, product, or process disclosed, or represents that its use would not infringe privately-owned rights. Idaho National Laboratory is a multi-program laboratory operated by Battelle Energy Alliance LLC, for the United States Department of Energy under Contract DE-AC07-05ID14517.

\section{REFERENCES}

[1] I. Humar, M. Gradišar, T. Turk, "The impact of color combinations on the legbility of web page text presented on CRT displays," International Journal of Industrial Ergonomics, vol. 38, pp. 885-899, 2008.

[2] I. Humar, M. Gradišar, T. Turk, "The impact of color combinations on the legibiltiy of text presented on LCDs," Applied Ergonomics, vol. 45, pp. 1510-1517, 2014.

[3] Human-System Interface Design Review Guidelines, NUREG-0700, Rev. 2, 2002.

[4] R. B. Kline, "Beyond significance testing: Reforming data analysis methods in behavioral research," American Psychological Association, 2004

[5] A. Arditi \& J. Cho "Letter case and text legibility in normal and low vision," Vision Research, vol. 47, pp. 2499-2505, 2007.

[6] Department of Defense Design Criteria Standard: Human Engineering, MIL-STD-1472 Rev. G, 2012

[7] H. Cai, P. A. Green, \& J. J. Kim, "Estimating the legibility of a single letter E viewed at different display angles," Applied Ergonomics, vol. 44, 2013.

[8] R. L. Boring, J. C. Joe, T. A. Ulrich, R. Lew, "Early-stage design and evaluation for nuclear power plant control room upgrades," Proceedings of the Human Factors and Ergonomics Society Annual Meeting, vol. 58, pp. 1909-1913, 20 